\title{
Doubling the dose of inhaled corticosteroids during asthma deterioration did not improve asthma control
}

Harrison TW, Oborne J, Newton S, et al. Doubling the dose of inhaled corticosteroid to prevent asthma exacerbations: randomised controlled trial. Lancet 2004;363:271-5.

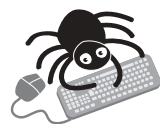

This article

contains extra text on the EBM

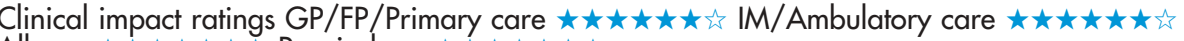
website

In patients with asthma, does doubling the dose of inhaled corticosteroid (ICS) reduce the need for prednisolone when control starts to deteriorate?

METHODS

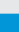

Design: randomised, placebo controlled trial.

Allocation: concealed.*

Blinding: blinded (clinicians and patients).*

Follow-up period: 12 months.

Setting: a hospital in Nottingham, UK.

Patients: 390 patients who were $\geqslant 16$ years of age (mean age 49 y, $67 \%$ women) with a clinical diagnosis of asthma; were taking ICS, 100-2000 $\mu \mathrm{g} / \mathrm{d}$ on a regular basis; and required oral corticosteroids or doubled their dose of ICS in the previous 12 months. Exclusion criteria: history of smoking $>10$ pack years or unstable asthma during a 2 week run in period.

D Intervention: patients were stratified by entry ICS dose (low to moderate or high) and allocated to an active inhaler $(n=192)$ or a placebo inhaler $(n=198)$. Study inhalers were matched to patients' regular ICS, type of inhaler, and dose. Patients were instructed to take their study inhaler for 14 days in addition to their usual treatment if their morning peak flow decreased by $15 \%$ from the mean peak flow, or if their daytime symptom score increased by 1 point on a 4 point scale from the median symptom score during the run in period. Patients recorded their peak flow and symptom scores for 28 days during which time they were started on 10 days of prednisolone, $30 \mathrm{mg} / \mathrm{d}$, if asthma control deteriorated to a point at which they would normally start oral corticosteroids, or peak flow decreased by $40 \%$.

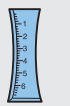

Outcomes: need for prednisolone. Secondary outcomes were maximum decrease in peak flow and increase in symptom scores while receiving the study inhaler.

Patient follow-up: $91 \%$ (intention to treat analysis).

*See glossary.

\section{MAIN RESULTS}

207 patients (53\%) started the study inhaler (54\% active and $46 \%$ placebo). Groups did not differ for need to start prednisolone, independent of whether they required the study inhaler (relative risk

For correspondence: $\mathrm{Dr} \mathrm{T} \mathrm{W}$ Harrison, Nottingham City Hospital, Nottingham, UK. tharris2@ncht.trent.nhs.uk

Sources of funding: NHS Executive. Active and placebo corticosteroid inhalers provided by AstraZeneca, GlaxoWellcome, 3M, and Baker Norton. reduction $5.5 \%, 95 \%$ CI -26 to 45 ; see table on website). Groups also did not differ for maximum decrease in mean peak flow or increase in symptoms scores while on the study inhaler (see table on website). The results were similar in patients taking a low to moderate ICS dose.

\section{CONCLUSION}

Doubling the dose of inhaled corticosteroids when asthma control begins to deteriorate did not reduce the need to start prednisolone and did not affect change in mean peak flow or asthma symptom scores.

Abstract and commentary also appear in ACP Journal Club.

\section{Commentory}

CSs are a mainstay of treatment for most patients with asthma. Although current guidelines recommend doubling the dose of ICS for mild asthma exacerbations, this simple intervention had not been investigated in properly designed studies. ${ }^{1}$ Harrison et al probed this recommendation in this randomised controlled trial. Although thoughtful in design, the study fails to provide conclusive evidence. Why?

Firstly, power is an issue. The authors claim the benefit of a large trial. However, in their calculation of sample size with systemic steroid use as primary endpoint, from doubling ICS they assumed a relative risk reduction (RRR) of $40 \%$ - a very large effect. Their sample size of 390 patients also was based on a projected control event rate that was $28 \%$ higher than actually observed (12\%). Thus, the study may have been too small to detect a smaller, yet important effect. Secondly, the results of what the authors described as per protocol analysis, an analysis in which practicing clinicians might be interested, deserve a second look. In patients who actually had to use the study inhaler because of worsening asthma, the active treatment resulted in a $20 \%$ RRR corresponding to a $6 \%$ absolute risk reduction $(p=0.53)$ for use of prednisolone. Furthermore, one might speculate whether other, perhaps more responsive outcome measures such as an asthma quality of life questionnaire would have shown a benefit. Finally, although the investigators instructed patients not to change their usual asthma management, no information is provided about actual use of other interventions that could have affected the results.

For clinical trialists in respirology, this study emphasises the need for adequately powered trials. However, it makes an important contribution by underscoring that guidelines should be evidence-based. If ICSs are truly ineffective in this setting, clinicians and patients would have to consider other therapies for asthma control (eg, omalizumab ${ }^{2}$ ). One hopes that their cost becomes less prohibitive.

Holger J Schünemann, MD, PhD University at Buffalo, State University of New York Buffalo, New York, USA

1 National Asthma Education and Prevention Program. Practical guide for the diagnosis of management of asthma. Bethesda, MD: National Institutes of Health; 1997. www.nhlbi.nih.gov/health/prof/lung/asthma/practgde.htm

2 Walker S, Monteil M, Phelan K, ef al. Anti-lgE for chronic asthma. Cochrane Database Syst Rev 2004;(3):CD003559. 